\title{
Modeling of an Organizational Environment by System Dynamics and Fuzzy Logic
}

\author{
Hafida Bouloiz $^{1}$, Emmanuel Garbolino ${ }^{2}$, Mohamed Tkiouat ${ }^{3}$ \\ ${ }^{1}$ Industrial Engineering Department, National School of Applied Sciences, ENSA, \\ University Ibn Zohr, Agadir, Morocco \\ ${ }^{2}$ Crisis and Risk Research Centre, Mines ParisTech, Rue Claude Daunesse, Sophia Antipolis, France \\ ${ }^{3}$ Industrial Engineering Department, Engineers' Mohammadia School, Rabat, Morocco \\ Email: h.bouloiz@ensa-agadir.ac.ma, emmanuel.garbolino@mines-paristech.fr, tkiouat@emi.ac.ma
}

Received May 14, 2013; revised June 14, 2013; accepted June 21, 2013

Copyright (C) 2013 Hafida Bouloiz et al. This is an open access article distributed under the Creative Commons Attribution License, which permits unrestricted use, distribution, and reproduction in any medium, provided the original work is properly cited.

\begin{abstract}
This paper presents an analysis, by a combination of dynamic systems and fuzzy logic, of the work environment of human factor. This environment contains a set of factors (variables) that influence human behavior in the context of industrial safety. The definition of these behavioral factors is made from a prior synthesis of the main factors that influence the safe behavior of human factor. The use of system dynamics allows identifying causal interactions between all these factors and representing these interactions through a systemic and dynamic vision. Fuzzy logic is used to account the qualitative and uncertain nature of variables value (called linguistic value) resulting from the phenomenon of perception. This work is a new conceptualization of dynamic systems and fuzzy logic for modeling the safe behavior of human factor.
\end{abstract}

Keywords: Human Behavior; System Dynamics; Fuzzy Theory; Dynamic Modeling; Systemic Approach

\section{Introduction}

Behavior of human factor is an important key in industrial safety. The results of the lessons learned from the accidents show that almost two thirds of the accidents are caused by behavior problems (procedural violations, errors...). Another reason to be interested in the behavior of operators is that the behavior is a major lever of safety in addition to technical and organizational improvements.

In [1] the authors showed that the human factor is in interaction with all components of the system through functions (formalized with the use case diagram) carried out by the operators. This network of interactions within the system shows that the human factor in addition to the organizational environment defines its behavior by avoiding any situation of risk (Figure 1).

Consideration of human factor and the organizational environment in risk management appears to be a key element to improve industrial safety [1]. This environment contains multiple factors that influence human behavior. Analysis of this situation leads to more understand the complexity and the diversity of the problem, due to several factors (psychological, psychosocial, or- ganizational and also economical). In addition, the human behavior cannot be assessed in a qualitative manner with precision and certainty.

The complexity of this problem, the existence of interactions, and the difficult nature to quantify factors influencing human behavior, all of these have led us to define a modeling approach to allow a detailed analysis of the main factors explaining the performance of human factor and causal interactions that link these factors, and to establish a decision support allowing to identify the principles of action to develop and channel operators' behavior in the context of industrial safety.

This paper contributes to the modeling of safe behav-

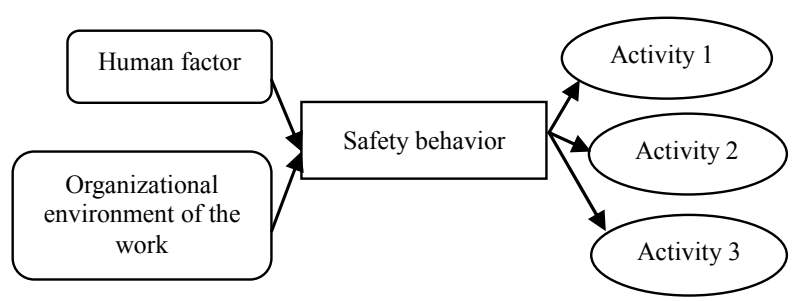

Figure 1. Relation between human factor and the activities of a system. 
ior of human factor, in which we combined several techniques that we seemed able to deal the problem. These techniques are:

- System dynamics used on the one hand as a technique to describe the different variables of the system and to analyze the causal interactions between these variables, on the other hand as a method of reflection and action. Indeed, system dynamics is based on methods which make it possible to study complex systems with a systemic vision. It is a way of thinking, because it allows analyzing the causality within a system "systemic character", and a mode of action, since it is part of an approach of decision support.

- the theory of fuzzy sets used to account for the qualitative, uncertain and linguistic character of the variables (in this text, variables are behavioral factors) influencing safe behavior of human factor.

This paper is organized as follows: First of all, we specify the main criteria of safe behavior of the human factor. After, we describe the principle of the proposed generic model of safe behavior founded on the system dynamics and the theory of fuzzy sets. This step led to a thorough investigation followed by dynamic modeling using the concepts of fuzzy sets. This work has made a generic simulation, in which the value of variables was conducted through interviews with employees of a chemical industry in Morocco.

\section{The Main Criteria of Safe Behavior of Human Factor}

Several definitions of the concept "behavior" exist in the literature. According to [2], behavior is "the set of reactions objectively observable that an organization will typically have a nervous system performs in response to stimulation of the environment, themselves objectively observable". In the [3], the behavior is defined as "a reality apprehended as observation units, acts, in which the frequency and the sequences are susceptible to change; it translates into action the image of the situation as it is developed, with its own tools: the behavior expresses a form of representation and construction of a particular world". According to [4] there are two types of safety behavior. The first one corresponds to the caution, which consists for example to respect and implement several safety rules, to wear protective equipment, etc. the second type is safe initiative or behavior of initiative for safety [5]. This second type of behavior consists to make suggestions in order to improve safety or to take initiative to avoid a situation of risk or also to propose corrective measures when equipment is defective. Transgressions and violations of rules, non-compliance procedures are the behaviors to avoid. The right strategy is to promote in the same time both types of safety behavior, namely caution and safety initiative. Several works have studied behavior of human factor. These works studies the behavior either from a single aspect: competence [6], personality $[7,8]$, performance [9], or they presents psychological or psychosocial factors influencing behavior: stress $[10,11]$, motivation $[12,13]$, fatigue $[10,14]$, precision in the work [15], feeling self-efficacy [16], intelligence, emotionality [17], conflict $[18,19]$. Other socioorganizational factors have been the subject of research showing the influence of these factors on the behavior of operators. In the [5], the authors show that the microorganizational factors such as degree of internal cohesion of the work group, degree of cooperation of the group and the supervisor are potential determinants of the propensity of operators to be cautious and to have safe initiative. Other studies [20] show that the macro-organizational factor, such as the degree of commitment of managers in prevention, is an important factor that positively influences the safety behavior of workers. Some researchers were interested to study the causal interactions between psychological factors influencing the behavior of workers. But these interactions are presented individually, for example, the impact of variation of the effort on the performance [21], the effect of motivation on the decision to act [12]. A generic model of behavior has been proposed by [22]. His model presents causal interactions between psychological factors (motivation, stress, performance, satisfaction, feeling self-efficacy) and psychosocial factors (conflict, communication), in order to define a model to study the effects of these two factors on the efficiency of operators in a production system.

\section{Proposal of Generic Model}

\subsection{Principle of a Generic Model of Safe Behavior of Human Factor}

In the proposed model, we consider the psychological, psychosocial, organizational, and also economic factors in order to develop a representative model of safety behavior.

The objective is not to study exhaustively all the factors influencing safety behavior, but to give a representative model, precise and complex. The selected factors are factors that are designed to analyze human behavior mainly in the workplace and have been objects of scientific studies. The definition of causal interactions between these factors is also based on these scientific studies.

\subsection{Modeling Methods}

\subsubsection{System Dynamics}

System dynamics is the theory of system structures, a theory that deals with the study of the causal interactions between the components which constitute the structure of a complex system. It is a modeling methodology for understanding and representing complex systems and ana- 
lyzing their dynamic behavior [23]. It finds its origin in cybernetics, which is the interdisciplinary study of the structure of regulatory systems [23]. System dynamics is a modeling method that allows a system to be represented in terms of feedback. It is founded on the original work of Forrester, who defined it as "the investigation of the information-feedback character of industrial systems and the use of models for the design of improved organizational form and guiding policy" [24]. System dynamics deals with the study of how the behavior of complex system changes over time [23].

System dynamics has two interesting aspects: systemic study of the concept of feedback and dynamic study of system behavior. It shows how the structure of a feedback system and the loops that it contains are responsible for its dynamic behavior. It is a method that focuses on the interactions between structural components, and behavior that is founded on the concept of feedback [25]. It is a methodology for designing and analyzing a system and simulating its behavior. According to [26] the syntax of system dynamics is neutral and independent of the field of application.

The model of system dynamics consists of three types of element (Figure 2): stock (or level) elements (also called state variables); flow elements; and auxiliary variables and constants [27].

\subsubsection{Theory of Fuzzy Sets}

The theoretical basis of fuzzy logic (fuzzy logic) has been established in early 1965 by Professor Zadeh University of California, Berkeley. This technique combines the concepts of "fuzzy subset" and "possibility theory" [28]. It is an approach based on human reasoning rather than on difficult calculations [28].

The theory of fuzzy sets offers a formal framework to model the imprecise and uncertain aspects of natural language. According to Zadeh [29-31], this theory is the most suitable formalism to describe qualitatively the linguistic variables.

This approach allows understanding natural phenomena which are imprecise and difficult to model based on the definition of rules and membership functions in sets called "fuzzy sets" [28]. Its interest is related to the for-

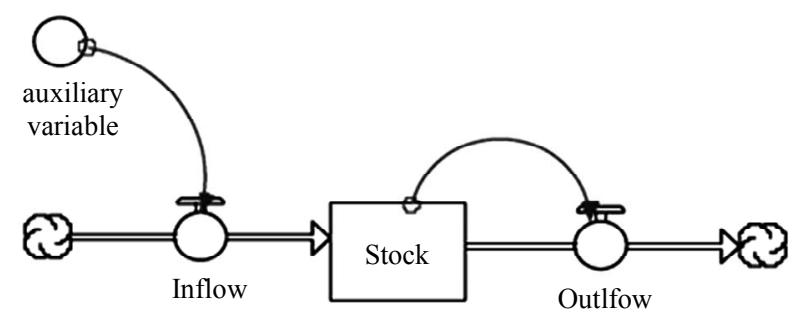

Figure 2. Schematic of a system dynamics model using the stock, flow and auxiliary variables proposed by Forrester (Forrester, 1961). malization of linguistic variables which are used to model imprecise or vague knowledge about a variable whose value can be unknown as low stress or low motivation. In order to represent these variables linguistically, the fuzzy sets are used.

A fuzzy set is completely defined by the data of its membership function. From such a function, a number of characteristics of fuzzy subset can be studied.

A linguistic variable is a triple $(\mathrm{V}, \mathrm{X}, \mathrm{Tg})$, where $\mathrm{V}$ is a variable defined on a reference set $\mathrm{X}$. The overall $\mathrm{Tv}=$ $\{\mathrm{A} 1, \mathrm{~A} 2, \ldots\}$, finite or infinite, contains standardized fuzzy subsets of $X$, used to characterize $V$. The number of elements of $\mathrm{Tv}$ is greater or less. Figure 3 shows an example of a representation of the linguistic variable "motivation." In this example, $\mathrm{V}=$ Motivation $\mathrm{X}=\mathrm{R} ; \mathrm{Tv}$ $=\{$ low, high $\}$.

In fuzzy logic, the fuzzy reasoning, also known as approximate reasoning, based on fuzzy rules which are expressed in natural language using linguistic variables [28].

\subsection{Representation by a Causal Diagram}

We recall here that the most existing studies that have studied the causal interactions between factors, influencing human behavior, have studied these interactions individually. Thus, we aggregated these individual causal interactions in order to develop a generic model of safety behavior. The use of existing scientific works enables us to give credibility to our model. Figure $\mathbf{4}$ presents the causal diagram of behavior developed under software VENSIM.

This diagram shows a set of causal interdependencies between factors influencing the safe behavior of operators. To structure the presentation and the explanation of this diagram, we divide these factors into two categories. The first category corresponds to factors that directly influence the safety behavior. These factors are: precision, feeling self-efficacy, intelligent conduct, motivation, stress. The second category corresponds to the factors that influence factors of the first category. Gradually, as we present each factor of the first category, we also explain the factors of the second category

\subsubsection{Description of Factors Influencing Safety Behavior of Operators \\ 1) Motivation}

Motivation is an important behavioral factor that di-

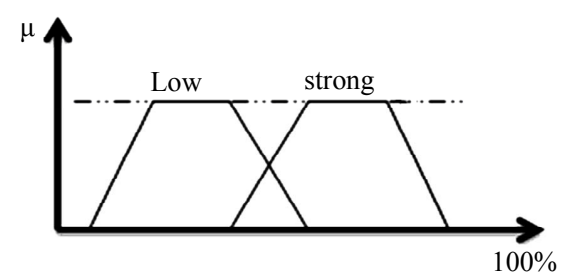

Figure 3. Linguistic variable "motivation”. 


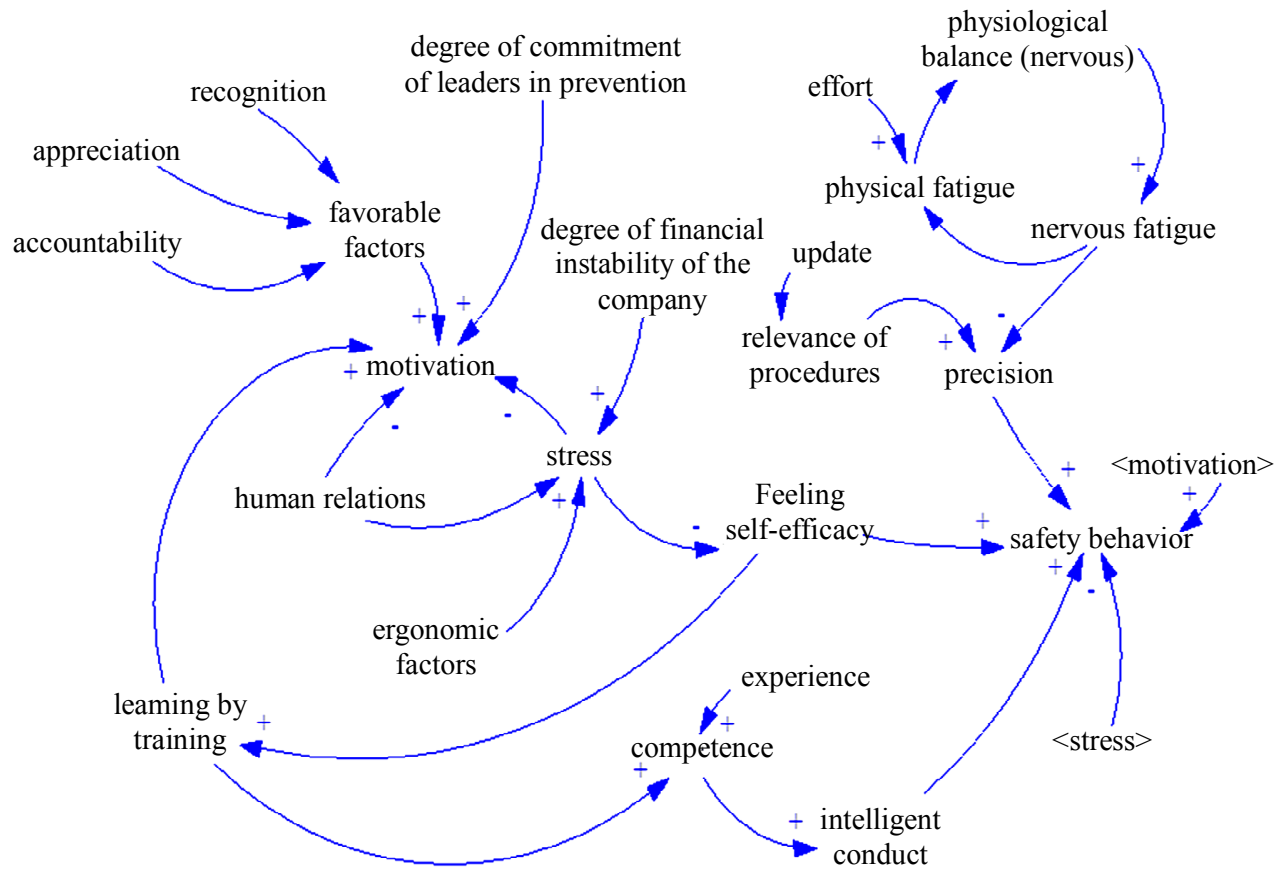

Figure 4. Generic causal diagram of safety behavior.

rectly influences the decision to act. This factor has been the subject of several studies and the many theories have been developed around this factor [32-38].

Motivation is the hypothetical constructs used to describe the internal and/or external forces producing release, direction, intensity and persistence of behavior.

In [12] the authors are interested to develop a more detailed model of motivation based on system dynamics. Their studies consist to explain why a person subject to a change in his environment will actually decide to act (action). They call "motivation" the process that leads to action and to decide.

Several factors influence the motivation process. We are not trying to be exhaustive but we limit only to some of these factors. Researches show the presence of a strong link between improving human relations and motivation of operators [39].

Aggression and sanctions are factors that increase stress and demotivate operators. While recognition and appreciation of their work increases their motivation. Similarly, accountability is an important factor influencing employee motivation. It results in the freedom of decision and action of employees. Training is also a source of motivation which increases their commitment to a specific activity. In [20], the author shows that organizational factor such as degree of involvement of manager in the health and safety in the work has a positive influence on the safety behavior of workers. This is a factor influencing worker motivation.

\section{2) Stress}

The workplace remains a major source of psychologi- cal stress [40]. Stress is one of the factors studied to analyze the behavior of the human factor in the work. For psychologists, stress is the result of any emotional, physical, social, economic or other which requires a response or change in a specific situation. In [41] the authors presented stress as a process in three major conceptual phases, themselves influenced by personal factors, social and environmental. These phases are: stressors, stress and consequences [42].

Stressors are the source of stress existing in the work environment. Stress, the second phase of the stress process can be long or short term depending on the nature of stressors. This second phase constitutes psychological interpretation and experience of events seen as stressful by an individual. Finally, the consequences, which are behavioral manifestations, psychological, physiological and organizational events, results the prolonged stress.

In the [21], the author presented a stress model using system dynamics. His model consists to develop causal interactions between stress and some psychological factors such as: motivation and mood.

Human relationships are a source which influences stress. Aggression increases stress of operators. The working environment is also stressful. Factors of this environment are ergonomic factors which exert pressure on the person such as: noise, vibration, temperature... etc.

Another factor that seems interesting to introduce it as a factor influencing stress is economic factor corresponding to the degree of instability of the company in the financial market. A recent investigative study shows the influence of the financial crisis mainly on workers stress 
[43].

\section{3) Feeling self-efficacy}

Feeling self-efficacy is an important factor that directly influences the behavior of operators. According to cognitive theory [44], feeling self-efficacy constitutes the belief that an individual has in its ability to produce or not a task. Confidence that the person up in his capacities to produce desired effects influences their aspirations, their choices, their vulnerability to stress and their level of perseverance [44]. This confidence has a logical link with learning through training which influences motivation [45].

\section{4) Precision in the work}

Precision in the work is related to the quality and efficiency of activity achieved by the operators. This is a factor that leads to the presence or absence of errors, and therefore directly influences the behavior of operators.

In [22] the author presented a model of imprecision in the work. Several factors affect the accuracy or inaccuracy in the work. These include: fatigue. According to [46], fatigue is a decrease in activity of a living system and related to the activity of this system. A simple loop appears as soon as we analyze the physiological phenomenon of fatigue [14] (Figure 5).

This loop is positive and can become dangerous in case of nervous breakdown. According to [47], nervous fatigue is manifested by an inability to maintain a system with an initial devolution accompanied by increased errors and reduced vigilance. The relevance of procedures is an important factor that increases the precision at work, while the absence or the incorrect information leads to imprecision.

\section{5) Intelligent conduct}

According to psychologists, intelligent conduct corresponds to a mental level which is concretized by the behavior, the adaptation, the ease of solving problems, and the ability of an operator to predict the effects of his conduct. Intelligent driving is associated with competence.

In the [48], the authors show that the competence is defined as a result of the use of internal expertise in an external context: Individual competence $=$ processing mechanisms + internal expertise + external context.

Modeling of competence was studied by several research which proposed models of competence $[49,50]$ [51]. In the [52] the authors proposed a model of competence that distinguishes knowledge, expertise and skills.

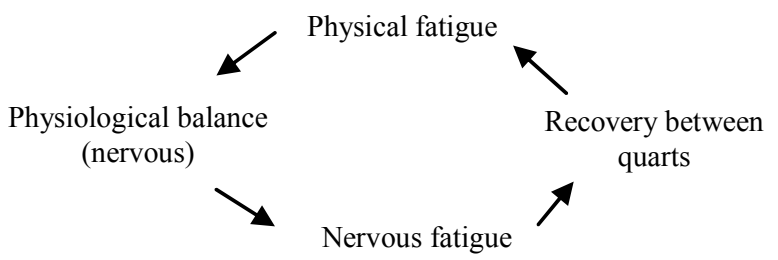

Figure 5. Loop of fatigue.
Competence can be seen as a process but also as a disposition to act [53].

Competence is a process which makes it possible to build or to adapt strategies for action by mobilizing the resources needed to accomplish a mission. Learning through training is an important source of competence development [54] and a powerful tool able to develop safety behavior [55].

Experience is also an important source of learning and consequently affects competence. According to [56], an individual "applies his prior knowledge, those created by his perceptual experiences and those made by his culture".

\subsubsection{Model Simulation}

Any operation simulation in system dynamics begins from a specified state of the system. Before running the simulation, the initial conditions for each state (or stock) variable must be defined.

The estimated value of the variables was conducted through interviews with employees of a chemical industry in Morocco. The method consists to develop an evaluation grid with a qualitative value scale (very low, low, medium, strong, very strong) which corresponds to the interval $[0 ; 0.25 ; 0.5 ; 0.75 ; 1]$. A value of 0 means very low and the value 1 means very strong. This grid is then presented to the subjects interviewed in order to give, in linguistic terms, an estimate of each variable. Each linguistic term is identified with a fuzzy subset as we will see in the next section.

Various sensitivity, or case studies, such as examining changes in the different variables, could be carried out using the proposed model. We present an example of a case study corresponding to loop of motivation.

The following table shows the simulation conditions of this case study.

The values given for the current situation correspond to the values of variables estimated through the interview. The duration of the simulation period is defined arbitrarily as six days.

Figure 6 presents the simulation results of the case study given in Table 1.

This figure shows clearly the influence of motivation on the behavior of operators. Indeed, as shown in the diagram causal (Figure 4), several factors (or variables) influence motivation such as: degree of commitment of managers in prevention, human relations, and still others factors such as recognition, appreciation and accountability. A disturbance in these factors affects the behavior of operators.

\subsection{Fuzzy Model Representation}

\subsubsection{Justification for the Use of the Theory of Fuzzy Subsets}

The development of causal diagram and description of 


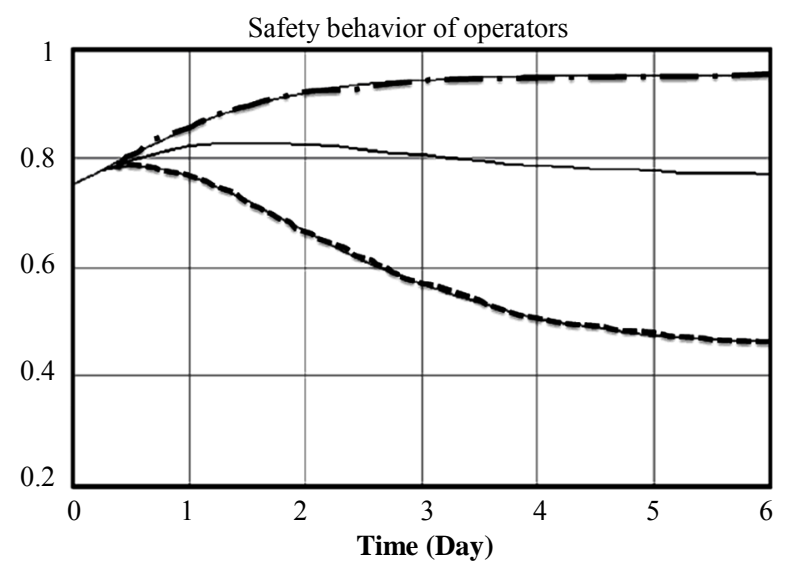

motivation: $+20 \%$ of motivation $\_$motivation: $-20 \%$ of motivation Safety behavior: current

Figure 6. Effects of motivation on human behavior.

Table 1. Case study conditions.

\begin{tabular}{ccc}
\hline Case Study & \multicolumn{1}{c}{ Data Set } & Description \\
\hline Motivation & Current situation & 0.75 \\
Time bounds for model & Low motivation & $\begin{array}{c}\text { Degree of increase: }+20 \% \text { of } \\
\text { the current situation } \\
\text { Degree of decrease: }-20 \% \\
\text { of the current situation } \\
\text { Initial time: } 0 \text { Final time: } 6\end{array}$ \\
Time step & 0.25 day \\
\hline
\end{tabular}

causal interactions between the main behavioral factors have shown how and why it was interesting to analyze such problem with the system dynamics. They also showed that the proposed causal model uncorked on linguistic variables whose value is mainly due to the phenomena of perception. Each linguistic term is identified with a fuzzy subset as we will explain.

But the translation of these linguistic values into digital values raises major questions. For example, when the motivation takes the value of 0.75 , using natural language, we say that the motivation is high. What it means the motivation of operators is high? How to represent this linguistic value? How to formulate the quantification language? And finally, how to integrate these linguistic values in a system? We have also encountered another problem in the evaluation of some variables. There were answers that begin with "I think", for example: "I think" the level of commitment of managers in prevention is low, I think the level of human relationships is high... etc., which gives uncertain nature of the values of these variables.

Therefore, we have recognized imprecise (i.e. strong, weak, etc.) and uncertain (responses that begin with "I think") character of these variables. In order to find the answers for these questions, the new concept of set-membership of a gradual element to a set appears as an ideal model for behavioral factors. Each linguistic term is therefore identified to a subset.

\subsubsection{Application to a Causal Model of Behavioral Factors}

The identification of causal relationships has been well defined in the first part of the proposed approach. The definition and description of these interactions has allowed us to estimate the strength of each interaction in a loop of the model. The method consists to break all feedback loops of the model in an individual interaction sequence. Figure 7 shows an example of such sequence:

The estimate of the intensity of each sequence of interaction is collected in a matrix in which each axis contains linguistic values. Each linguistic expression is identified as a fuzzy subset. Figure 8 shows an example of estimation matrix of strength of the causal interaction between variables in the example of the sequence shown above.

This matrix is then interpreted as an interaction. The value of this interaction is constructed from linguistic expression that the estimation in the matrix suggests. In the example of Figure 7:

- If the degree of commitment of the managers in the prevention is very low then the motivation is very low;

- If the degree of commitment of the managers in the prevention is low then the motivation is very low or low;

degree of commitment of managers in the prevention

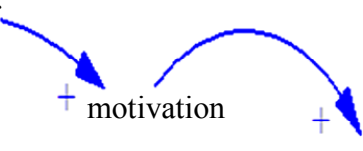

safety behavior

Figure 7. Example of a sequence of causal interaction.

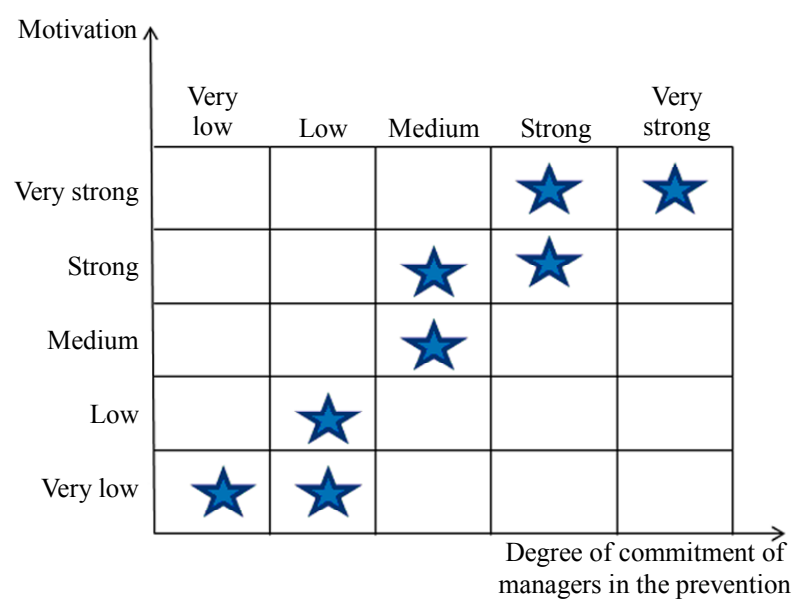

Figure 8. Example of estimation matrix of the strength of a causal interaction. 
- If the degree of commitment of the managers in the prevention is medium then the motivation is medium or high;

- If the degree of commitment of the managers in the prevention is strong then the motivation is strong or very strong;

- If the degree of commitment of the managers in the prevention is very strong then the motivation is very strong;

Similarly, we have estimated the intensity of each interaction in the proposed model. The evaluation of these interactions makes reference to the rules of computation in fuzzy logic.

\section{Conclusions}

In this work, we have developed a methodological approach for controlling the safe behavior of the human factor. It is an approach to decision support combining two techniques: system dynamics and fuzzy sets theory. This approach allows understanding the problem in its generality and studying a specific application.

System dynamics allowed modeling the complexity of the problem by analyzing the set of causal interactions (forming feedback loops) between several variables that influence human behavior. The practical significance of this technique is to identify the principles of action to develop and channel safety behaviors of operators. The model can be used as a support to assist managers to study the impact of a potential disturbance on the performance of the operators.

The theory of fuzzy sets allowed accounting for the qualitative and uncertain character of the variables in the model. The data obtained by the method of interviews allowed us to obtain data near the current language with linguistic expressions.

The methodology presented in this work is already being implemented. It will be developed in other case studies. Indeed, we will apply this methodology to a sample of industries of different activity in order to assess the safety behavior of operators in relation to the level of danger that exists in these industrial activities.

\section{REFERENCES}

[1] H. Bouloiz, E. Garbolino and M. Tkiouat, "Contribution of a Systemic Modeling Approach Applied to Support Risk Analysis of a Storage Unit of Chemical Products in Morocco," Journal of Loss Prevention in the Process Industries, Vol. 23, No. 2, 2010, pp. 312-322. http://dx.doi.org/10.1016/j.jlp.2009.12.001

[2] J. B. Watson, "Psychology as the Behaviorist Views It," Psychological Review, Vol. 20, No. 2, 1913, pp. 158-177. http://dx.doi.org/10.1037/h0074428

[3] A. Gallo, "The Animals, Psychology and Behavior," 1999 , p. 63.

[4] M. Simard and A. Marchand, "Adaptation of Supervisors to Participatory Management of Accident Prevention," Industrial Relations, Vol. 50, No. 3, pp. 567-586.

[5] M. Simard and A. Marchand, "Participation of the Workers in the Prevention of Occupational Accidents: Forms, Efficacy and Determinants," Report R-154, IRSST, Montreal, 1997, p. 40.

[6] M. Harzallah and F. Vernadat, "It-Based Competency Modeling and Management: From Theory to Practice in Enterprise Engineering and Operations," Computers in Industry, Vol. 48, No. 2, 2002, pp. 157-179. http://dx.doi.org/10.1016/S0166-3615(02)00003-9

[7] R. McCrae and John, "An Introduction to the Five-Factor Model and Its Applications," Journal of Personality, Vol. 60, No. 2, 1992, pp. 175-215.

[8] P. J. Howard, "The Owner's Manual for the Brain," Bard Press, 2000.

[9] P. Siebers, T. Baines, S. Mason and J. Ladbrook, "Modeling of Human Variation in Assembly Line Models," Proceedings of Operational Research Society Simulation Workshop (SW04), UK, 2004, pp. 515-526.

[10] N. Giambiasi, C. Frydman and M. Seck, "Modeling and Simulation of Human Behavior with the Devs Formalism," Proceeding of the 6th Conference of Francophone Modeling and Simulation, MOSIM'06, Morocco, 2005, pp. 1-8.

[11] R. Frere, “Contribution à L'intégration D'aspects Humains dans la Modélisation des Systèmes de Fabrication: Vers une Gestion Conjointe des Ressources Humaines and de la Production," Thèse de Doctorat, Université de Valenciennes and du Hainaut-Cambrésis, Valenciennes, 2000.

[12] M. Karsky and G. Donnadieu, "The Dynamic of Behavior and Motivation," 1990.

[13] R. Ashgate, Ryan and E. Deci, "Intrinsic and Extrinsic Motivations: Classic Definitions and New Directions," Contemporary Educational Psychology, Vol. 25, No. 1, 2000, pp. 54-67.

http://dx.doi.org/10.1006/ceps.1999.1020

[14] M Karsky and M. Adamo, "Application de la Dynamique des Systèmes and de la Logique Floue à la Modélisation d'un Problème de Postes en Raffinerie," Actes du Congrès de 1'AFCET. Edition Hommes and Techniques. Tome 2, 1977, pp. 479-491.

[15] A Belyavin and E. Farmer, "Modeling the Workload and Performance of Psychomotor Tasks," Proceedings of the Conference on Behavior Representation in Modeling and Simulation (BRIMS), Baltimore, 2006, pp. 1-16.

[16] A. Bandura, "Self-Efficacy: The Exercise of Control," Freeman, New York, 1997.

[17] A. P. Gemelli, "Human Factor: Accidents in the Work," 1955.

[18] A. Cornet, "Investigation of the Social Climate and Satisfaction in the Work: A Tool for Management of Human Resources," Technical Report, 13th Summer University, 1995.

[19] R. Frere, "Contribution to the Integration of Human As- 
pects in the Modeling of Manufacturing Systems: Towards Joint Management of Human Resources and Production," Ph.D. Thesis, University of Valenciennes, 2000, p. 230 .

[20] J. H. T. H. Andriessen, "Safe Behavior and Safety Motivation," Journal of Occupational Accidents, Vol. 1, No. 4, 1982, pp. 368-376.

[21] C. Jones, "Behavioral Theory in Simulation: Ambiguous Results from Simple Relationships," Proceedings the 23rd International Conference of the System Dynamics Society, 2005, pp. 1-25.

[22] S. Elkosantini, "Contribution to the Dynamic Modeling of Human Operators," Ph.D. Thesis, Blaise Pascal University, 2007

[23] J. Aracil, "Introduction à la Dynamique des Systèmes," Presse Universitaire de Lyon, 1984, p. 414.

[24] J. W. Forrester, "Industrial Dynamics," MIT Press, Cambridge, 1961, p. 464.

[25] M. E. Paté-Cornell, "A Post Mortem Analysis of the Piper Alpha Accident: Technical and Organizational Factors," Risk Analysis, Vol. 13, No. 2, 1999, pp. 215-232. http://dx.doi.org/10.1111/j.1539-6924.1993.tb01071.x

[26] N. Wiener, "Cybernetics or Control and Communication in the Animal and the Machine," 1948.

[27] J. M. Garcia, "Theory and Practical Exercises of System Dynamics," Universitat Politecnica De Catalunya, Barcelona, 2006.

[28] F. Dernoncourt, "Introduction to Fuzzy Logic," 2011, p. 14.

[29] L. A. Zadeh, "Fuzzy Sets," Information and Control, Vol. 8, No. 3, 1965, pp. 338-353. http://dx.doi.org/10.1016/S0019-9958(65)90241-X

[30] L. A. Zadeh, "The Concept of a Linguistic Variable and Its Application to Approximate Reasoning," Information Science, 1975, pp. 199-249.

[31] L. A. Zadeh, "Fuzzy Sets as a Basis for Theory of Possibility," International Journal Fuzzy Sets and Systems, Vol. 100, No. 1, 1978, pp. 3-28.

[32] A. H. Maslow, "A Theory of Human Motivation," Psychological Review, 1954, pp. 370-396.

[33] F. Herzberg and V. Charles, "Work and the Nature of Man," Modern Enterprise Edition, Paris, 1959.

[34] D. McClelland, "Théorie des Besoins," 1961.

[35] J. R Hackman and G. R. Oldham, "Motivation through the Design of Work: Test of a Theory," Organizational Behavior and Human Performance, Vol. 16, No. 2, 1976, pp. 250-279. http://dx.doi.org/10.1016/0030-5073(76)90016-7

[36] V. H. Vroom, "Work and Motivation," John Wiley \& Sons, New York, 1964.

[37] J. S. Adams, "Inequity in Social Exchange," In: L. Berkowitz, Ed., Advances in Experimental Social Psychology, Vol. 2, Academic Press, New York, 1963, p. 267.

[38] M. J. Apter, "The Experience of Motivation: The Theory of Psychological Reversals," Academic Press, 1982, pp. 378.
[39] A. P. Gemelli, "Human Factor: Accidents in the Work," 1955.

[40] A. Marchand, A. Demers and P. Durand, "Does Work Really Cause Distress? The Contribution of Occupational Structure and Work Organization to the Experience of Psychological Distress," Social Science and Medicine, Vol. 61, No. 1, 2005, pp. 1-14. http://dx.doi.org/10.1016/j.socscimed.2004.11.037

[41] P. Hart and C. Cooper, "Occupational Stress: Toward a More Integrated Framework," In: N. Anderson, D. S. Ones, H. K. Sinangil and C. Viswesvaran, Eds., Handbook of Industrial Work and Organizational Psychology, Vol. 2, Sage, London, 2001.

[42] S. Harvey, F. Courcy, A. Petit, J. Hudon, M. Teed, O. Loiselle and A. Morin, "Organizational Interventions and Psychological Health in the Work: A Synthesis of Approaches," Report, Institute de Research en Sante et Securitie au Travail (IRSST), Montreal, 2006.

[43] D. Autissier and F. Bensebaa, "Investigation. Impact of Financial Crisis on Life in the Company," Perceptions and Behavior of Employees, 2009.

[44] A. Bandura and P. Carré, "Self-Efficacy. Sens of Personal Efficacy. Collection 'Psuchological Openings'," 2002, p. 860.

[45] R. Foucher and L. Morin, "Sentiment d'efficacité Personnelle et Apprentissage Dans des Dispositifs Ouverts De Formation: Résultats de Cinq Recherches Empiriques," 2007.

[46] S. Scherrer, "Physiologie Du Travail," 1967.

[47] H. Allain, "Th Fatigue: Biology to Pharmacology," Technical Report, University of Rennes 1, 1999.

[48] W. Zachary, T. Santarelli, J. Ryder, J. Stokes and D. Scolaro, "Developing a Multi-Tasking Cognitive Agent Using the Cognet/Igen Integrative Architecture," Proceeding of 10th Conference on Computer Generated Forces and Behavior Representation, 2001.

[49] X. Boucher and P. Burlat, "Towards the Integration of Skills in the Performance Management of the Company," Journal Européen des Systèmes Automatisés, Vol. 37, No. 3, 2003, pp. 363-390. http://dx.doi.org/10.3166/jesa.37.363-390

[50] E. Bonjour and M. Dulmet. "Articulation Entre Pilotage des Systèmes de Compétences et Gestion des Connaissances," 1er Colloque de Gestion des Compétences et des Connaissances en Génie Industriel, Nantes, 12-13 Décembre, 2002, pp. 43-50

[51] A. Boumane, A. Talbi, C. Tahon and D. Bouami, "Contribution to the Modeling of Competence. MOSIM'06, 'Modeling, Optimization and Simulation Systems: Challenges and Opportunities'," Rabat, Morocco, 2006.

[52] M. Harzallah, F. Vernadat, "It-Based Competency Modeling and Management: From Theory to Practice in Enterprise Engineering and Operations," Computers in Industry, Vol. 48, No. 2, 2002, pp. 157-179.

[53] Le Boterf, "Construire les Compétences Individuelles et Collectives: La Compétence N'est Plus ce Qu'elle Était," 2004.

[54] M. Cesar, "The Process of Organizational Learning: To- 
wards New Ways of Learning and the Management of Competences," 2005.

[55] C. Denis-Remis, "Approche de la Maîtrise des Risques par la Formation des Acteurs," Thèse de Doctorat, Ecole des Mines de Paris, 2007.

[56] M. Jimenez, "Psychology of Perception," Flammarion, Paris, 1997. 